\title{
What Is the Best Surgical Approach for Bilateral Pulmonary Hydatid Cysts in Children?
}

\author{
Aram Baram \\ University of Sulaimani, Sulaymaniyah, Iraq \\ Email: aram.baramm@gmail.com \\ Received 20 February 2015; accepted 12 March 2015; published 18 March 2015 \\ Copyright (C) 2015 by author and Scientific Research Publishing Inc. \\ This work is licensed under the Creative Commons Attribution International License (CC BY). \\ http://creativecommons.org/licenses/by/4.0/ \\ (c) (i) Open Access
}

\begin{abstract}
Introduction: The most common site for hydatid cysts in children is the lungs while in adult hepatic cysts are predominant. Bilateral pulmonary involvement is relatively rare and its surgical management is poorly described in the current literature. Until now no definite consensus has been described in the literature for the ideal surgical approach in pediatric bilateral pulmonary hydatid cysts (BPHC). The aim of this prospective cohort study was to describe the problems encountered in treating pediatric BPHC by two different surgical techniques. Patients and Methods: Between June 2007 and June 2014, 60 children (group one $=31$, group two $=29$ ) with BPHC were operated on in our center. Group one included all children with BPHC operated by single session bilateral anterolateral mini-thoracotomy. Group two included all cases operated by two stage standard posterolateral thoracotomy at 21 days interval. Results: In group one (19 males and 12 females), the mean age was 8.9 years $(3.5$ - 17). In group two, there were 29 cases $(18$ males and 11 females), the mean age was 9.6 years $(2-17)$. There was no significant statistical difference in terms of the presentations and age distribution. The duration of surgery in group one was significantly shorter and the duration of hospital stay was significantly shorter as well (group one 3.6 days versus 4.6 days in the second group). Pain scale was not more in the first group as it was believed to be. Conclusion: We believe that single session bilateral anterolateral thoracotomy is a better approach than either one stage successive thoracotomies, median sternotomy or clamshell thoracotomy as it involves less postoperative pain and does not precipitate a decrease in the respiratory capacity.
\end{abstract}

\section{Keywords}

Bilateral Pulmonary Hydatid Cyst (BPHC), Bilateral Anterolateral Thoracotomy 


\section{Introduction}

Echinococcus (hydatid disease or hydatidosis) is the most widespread, serious human cestode infection in the world. It is a zoonosis that is transmitted from domestic and wild members of canine family [1] [2], who become infected after eating infected viscera and are the hosts of the small adult worms. Domestic animals such as sheep, goats, cattle and camels ingest Echinococcus granulosus eggs while grazing. Pulmonary hydatid cysts are more common in male children [3]. The most common site for hydatid cysts in children is the lung while adults mostly have hepatic cysts. Bilateral pulmonary involvement is relatively rare and it's poorly described in the current literature [3]-[7]. Furthermore, combined lung and liver cysts are more frequent in children than adults. Some studies assume that high elasticity and compressible tissue of lungs in children leads to faster growing of hydatid cysts in lungs than liver and by consequence the pulmonary cysts are larger in size at presentation in comparison to the hepatic ones [6]-[9]. The standard treatment for pediatric pulmonary hydatid disease is parenchyma-preserving surgery; the safest technique is controlled evacuation technique with cystotomy or cystotomy plus capitonnage especially in case with giant cysts (i.e. $>10 \mathrm{~cm}$ or occupying more than $50 \%$ of the hemithorax) [1] [4] [5] [8].

Until now no definite consensus described in the literature for the ideal surgical approach in pediatric bilateral pulmonary hydatid cysts (BPHC).

In this cohort study we prospectively followed up 2 groups of children with pulmonary hydatidosis that were treated at our center, and we compared the clinical presentation, 2 different surgical treatment, and postoperative outcome.

The aim was to describe the problems encountered in treating BPHC by two different surgical techniques only in pediatric population.

\section{Patients and Methods}

Between June 2007 and June 2014, 60 children with BPHC were operated on in our center. During that period totally 309 children were operated for pulmonary hydatid cyst. Only the cases with bilateral hydatid cyst were included in this review. The operative approach decided after discussion with the family by explaining both approaches advantages and disadvantages.

In either group the diagnostic workup composed of thorough history, physical examination, hematological investigations looking for eosinophilia if exists, chest X-ray was the standard method of diagnosis and chest, brain and upper abdominal CT scan was performed for all to confirm or exclude the extra pulmonary cysts (Figures 1-3).

These 60 children were divided in to two groups: Group one (31 cases) included all children with bilateral hydatid cyst were operated by single session bilateral anterolateral mini-thoracotomy at the fourth or fifth intercostal space (Figure 4). The pleural cavities were opened in succession usually starting with the more affected side (Figure 5, Figure 6). While group two (29 cases) included all cases operated by two stage standard posterolateral thoracotomy at 21 days interval. Epidural catheter, at the level Th7 \pm Th8, for prolonged postoperative analgesia (lidocaine (1\%) 1 Fentanyl (5 mg/ml) 1 adrenaline 1:200,000) was applied.

The symptoms, signs, localizations, and radiologic findings of the cysts and the indications, specifications, and results of the two operative methods were studied. In both groups the pleural space drained by single chest drain which was removed at 24 - 48 hours postoperatively.

In both groups, the classical principles of pulmonary hydatid cyst surgery were followed strictly. The operative field was isolated with Povidone-iodine for protection (Figures 6-8). Conservative parenchymal resection techniques were the methods of choice. No any lobectomy or wedge resection was performed and the residual cavity treated by capitonnage.

During the period of the study the long-term outcome was evaluated. Antihelmentic therapy started preoperatively (Albendazole) and it was continued up to 3 months postoperatively.

Fisher's exact test with Yates correction or the two-tailed Students' t-test was used for comparison between groups. Differences were considered significant if the $p$ value was less than 0.05 .

\section{Results}

In group one there were 31 cases (19 males and 12 females), the mean age was 8.9 years (3.5 - 17). The main 


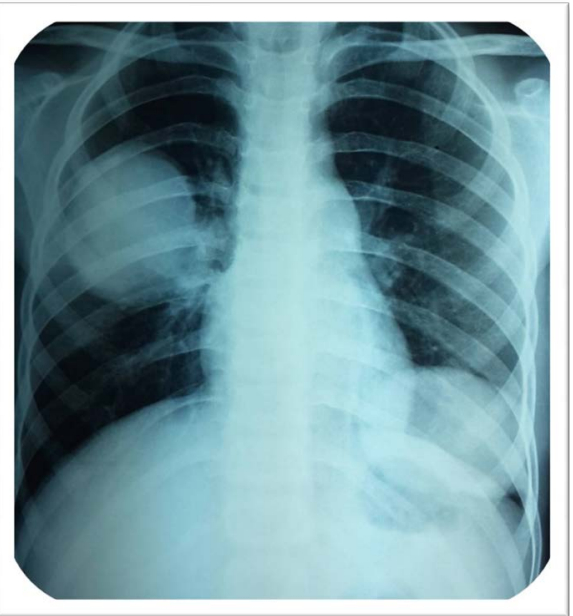

Figure 1. Bilateral intact pulmonary hydatid cysts.

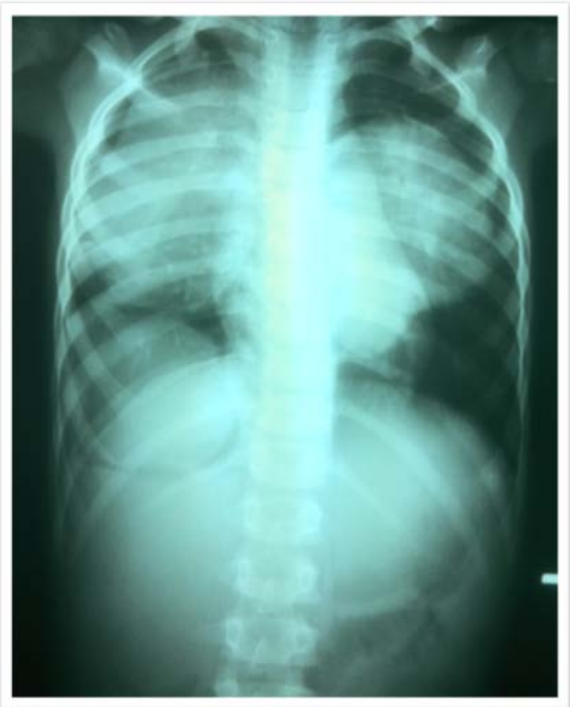

Figure 2. Bilateral giant pulmonary hydatid cysts.

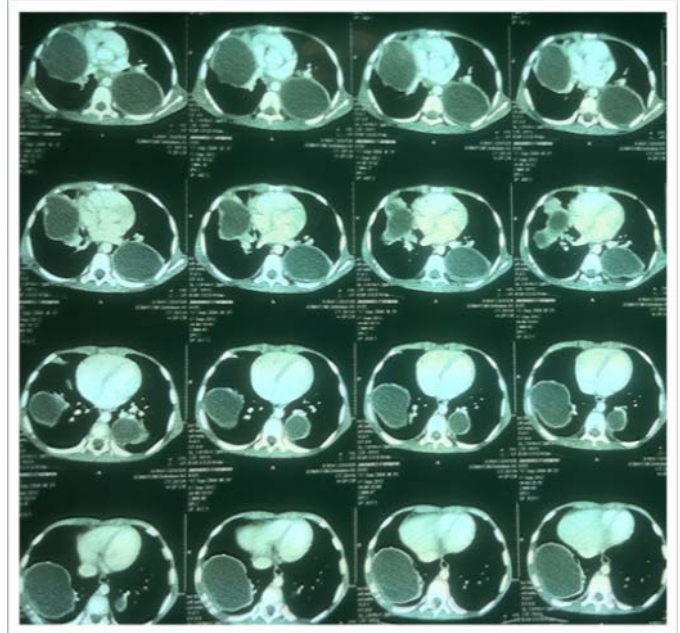

Figure 3. Chest CT scan shows bilateral multiple hydatid cysts. 


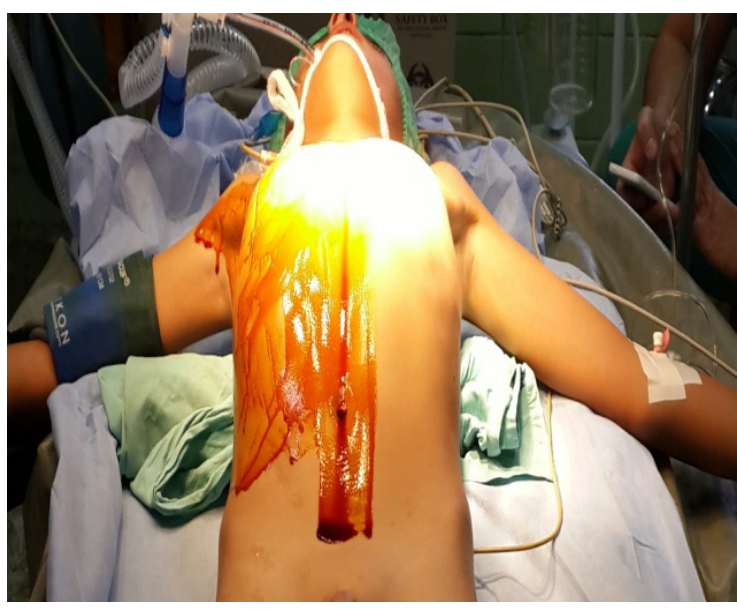

Figure 4. Patient's positioning for surgery.

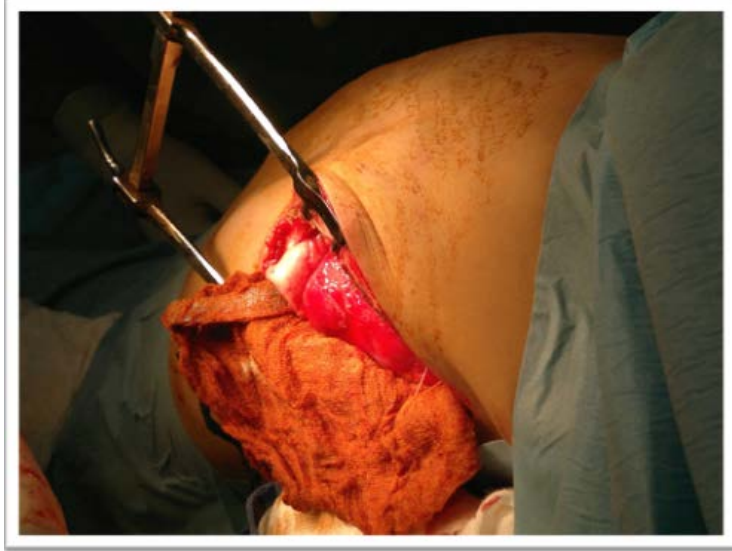

Figure 5. Cyst isolation for evacuation.

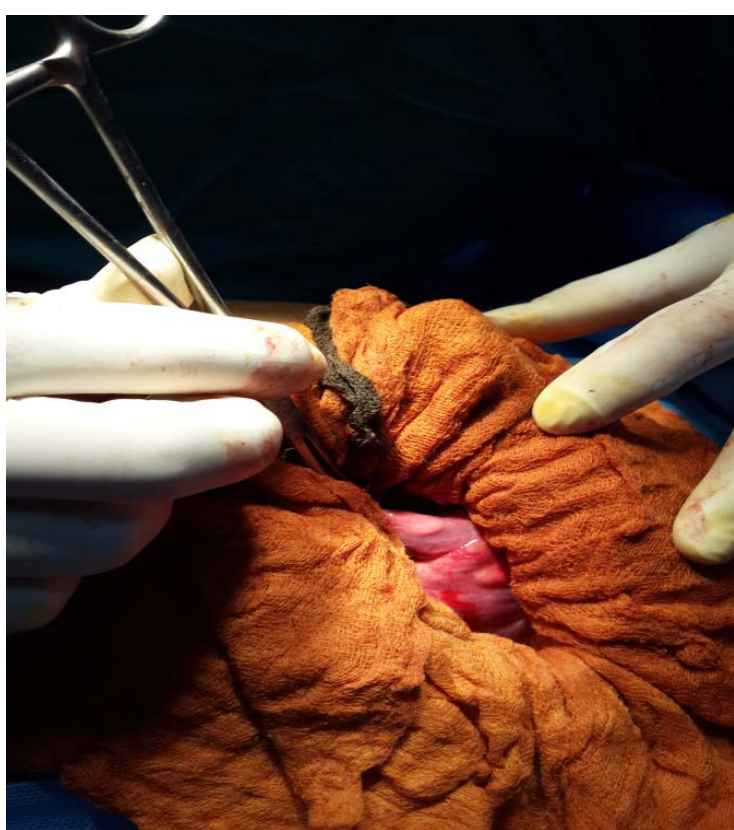

Figure 6. Strict isolation of the cyst to prevent any potential seeding. 


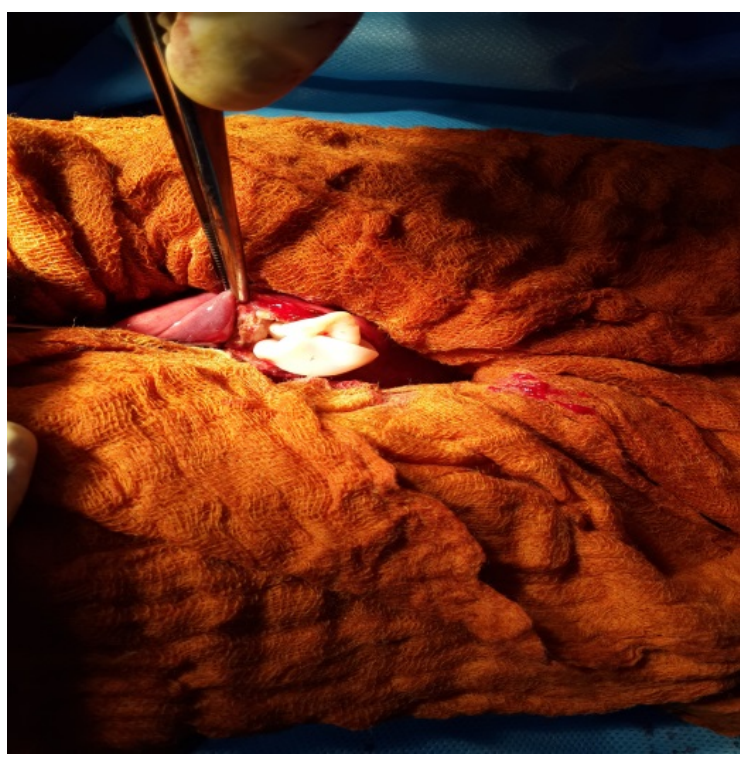

Figure 7. Cyst dilvered for excision.

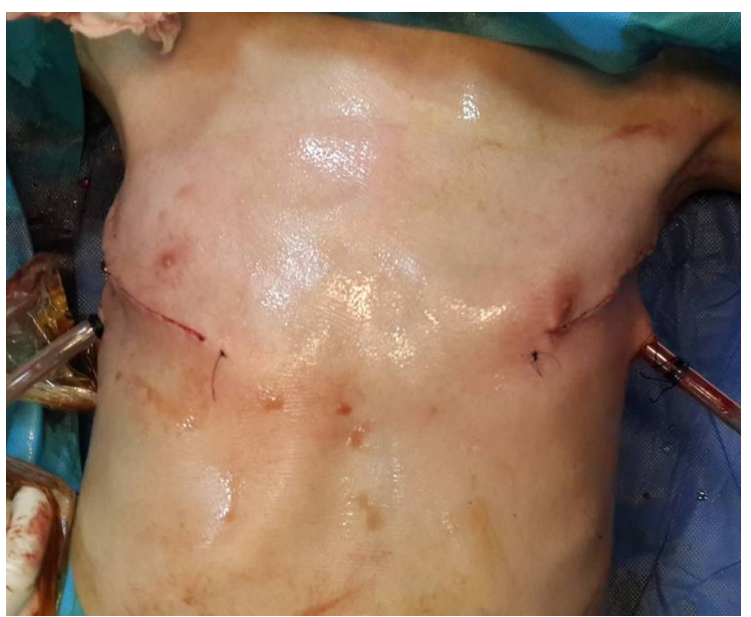

Figure 8. Final outcome two anterolateral incision.

presentation was accidentally found cysts (10 cases 32.25\%) on chest X-ray during investigation for non-specific chest symptoms. Other symptoms like chest infection (4\%), shortness of breath (4\%), chest pain (3\%), expectoration of cyst material (2\%), chronic cough (1\%), hemoptysis (1\%), weight loss (1\%) and ill health (1\%), Abdominal pain (1\%) and fever (1\%) were the presenting symptoms for the rest of the cases in the first group (Table 1, Table 2).

In group two, there were 29 cases (18 males and 11 females), the mean age was 9.6 years $(2-17)$. The main presentation was accidentally found cysts (11 cases $37.9 \%)$ on chest X-ray during investigation for non-specific chest symptoms. Other symptoms like chest infection (3\%), shortness of breath (3\%), chest pain (3\%), expectoration of cyst material (3\%), chronic cough (3\%), hemoptysis (2\%), weight loss $(1 \%)$ and ill health $(1 \%)$, Abdominal pain (1\%) and fever (1\%) were the presenting symptoms for the rest of the cases in this group (Table 1, Table 2).

Comparing these 2 groups we found no significant statistical difference in terms of the presentations and age distribution.

Totally 138 cysts were removed (70 in group one 68 in group two), in the first group there was 6 cases who had more than one cyst per lobe while in second group 4 cases had more than one cyst per lobe (Table 3).

We found a significant statistical difference for the duration of surgery in group one which was 92.4 minutes 
(60 - 180) while in group two it was 130.34 minutes (100 - 280). This shows statistically significant shorter duration of surgery for the first approach which may be associated with lower level of intra and postoperative morbidity (Table 2).

Duration of hospital stay was significantly shorter in the first group 3.6 days versus 4.6 days in the second group. This shows the superiority of the first approach in treatment of BPHC in pediatric population.

No significant statistical differences were noticed in the postoperative period. Pain was not more in the first group as it was believed to be. While other complications were nearly the same (Table 4).

Table 1. Clinical features of bilateral pulmonary hydatid cyst.

\begin{tabular}{|ccccccc}
\hline Symptoms \& Signs & \multicolumn{2}{c}{$\begin{array}{c}\text { Group 1 } \\
(\mathbf{n = 3 1 )}\end{array}$} & $\boldsymbol{p}$ value & \multicolumn{2}{c}{$\begin{array}{c}\text { Group 2 } \\
\text { (n= 29) }\end{array}$} & $p$ value \\
\hline Asymptomatic (accidentally found) & 10 & $32.25 \%$ & $p<0.0001$ & 11 & $37.9 \%$ & $p<0.0001$ \\
Chest infection & 4 & $12.9 \%$ & $p<0.0001$ & 4 & $13.8 \%$ & $p<0.0001$ \\
Shortness of breath & 3 & $9.6 \%$ & $p>0.0027$ & 4 & $13.8 \%$ & $p>0.0001$ \\
Chest pain & 3 & $9.6 \%$ & $p>0.0027$ & 3 & $10.34 \%$ & $p>0.0027$ \\
Expectoration of cyst material & 3 & $9.6 \%$ & $p>0.0027$ & 2 & $6.9 \%$ & $p>0.0445$ \\
Chronic cough & 3 & $9.6 \%$ & $p>0.0027$ & 1 & $3.24 \%$ & $p>0.0027$ \\
Hemoptysis & 2 & $6.45 \%$ & $p>0.0045$ & 1 & $3.24 \%$ & $p>0.0045$ \\
Weight loss and ill health & 1 & $3.22 \%$ & $p>0.3173$ & 1 & $3.24 \%$ & $p>0.3173$ \\
Abdominal pain & 1 & $3.22 \%$ & $p>0.3173$ & 1 & $3.24 \%$ & $p>0.3173$ \\
Fever & 1 & $3.22 \%$ & $p>0.3173$ & 1 & $3.24 \%$ & $p>0.3173$ \\
Total & 31 & $100 \%$ & & 29 & $100 \%$ & \\
\hline
\end{tabular}

Table 2. Patent's demographics and operation records.

\begin{tabular}{ccc}
\hline Patent's demographics and operation records & Group 1 & Group 2 \\
\hline Males & 19 & 18 \\
Females & 12 & 11 \\
Age & $(3.5-17)$ mean 8.9 & $(2-17)$ mean 9.6 \\
Duration of surgery in minutes & $(60-180)$ mean 92.4 minutes & $(100-280)$ mean 130.34 \\
Hospital stay & $(2-15)$ mean 3.6 days & $(2-8)$ mean 4.6 \\
\hline Table 3. Lobes affected. & & Group 2 \\
\hline Lobes & Group 1 & $18(26.47 \%)$ \\
\hline RLL & $22(31.42 \%)$ & $14(20.58 \%)$ \\
LLL & $20(28.57 \%)$ & $24(35.29 \%)$ \\
LUL & $12(17.14 \%)$ & $12(17.64 \%)$ \\
RUL & $9(12.85 \%)$ & $\mathbf{6 8}$ (100\%) \\
RML & $7(10 \%)$ & $\mathbf{7 0}(\mathbf{1 0 0} \%)$ \\
Total & &
\end{tabular}

Table 4. Postoperative complications.

\begin{tabular}{ccc}
\hline Postoperative complications & Group 1 & Group 2 \\
\hline Pain severity (Mosby scale) & $6-8$ & $6-8$ \\
Prolonged air leak & $2 \%$ & $2 \%$ \\
Pleural space complications & $0 \%$ & $0 \%$ \\
Chest infection & $1.5 \%$ & $1 \%$ \\
Wound infection & $0 \%$ & $0 \%$ \\
Systemic complications & $0 \%$ & $0 \%$ \\
Hospital stay & $(2-15)$ mean 3.6 days & $(2-8)$ mean 4.6 \\
\hline
\end{tabular}




\section{Discussion}

The lung is the second most commonly affected organ after the liver $10 \%$ to $40 \%$ of the cases [2] [3] [10]. Bilateral pulmonary hydatid (BPHC) varies from $4 \%$ to $26.7 \%$ [10]. Its surgical treatment includes different options for operative tactics and approaches, especially when it is associated with hepatic cyst. The advances in intensive care and the refinement of surgical skills have all contributed to the development of various new operative strategies and techniques in the treatment of BPHC.

Many authors recommend two-stage operations for BPHC with 2 - 3 weeks interval between the two interventions [11]-[13]. The rationale behind two-stage operations includes: 1) when lobectomy is required in the presence of pleural adhesions the single stage surgery is very difficult; 2) presence of an already poor cardiopulmonary reserve in bilateral cysts; and 3) pain is an important factor in determining the post-operative course of bilateral thoracotomy; 4) postoperative pulmonary complications may be difficult to manage [12] [14]. But nowadays these points can be dealt with safely in one stage surgery and they are no more an obstacle for one stage surgery.

In BPHC surgical treatment should be the first-choice treatment. Enucleation is not possible in ruptured cysts. Although methods which protect the parenchyma are preferred primarily, the necessity of large pulmonary resections in cases of pulmonary HC is still controversial [15]-[17]. In our two groups of patients, cystotomy-controlled evacuation was applied for all of the cases in either group. The parts of the cyst which were not surrounded by the lung parenchyma were excised as much as possible, but resection of healthy lung tissue was avoided even if adjacency with the cyst wall was present for the aim of protecting lung function as much as possible and all the residual cavities are treated by capitonnage as it is advised by most of the researchers [4]-[8].

Anatomical pulmonary resections are not recommended in BPHC because the lung tissue compressed by the cysts is generally intact and it re-expands after operation. In addition, there is a risk of recurrence in HC disease. Therefore, protection of lung parenchyma as much as possible is the golden rule in surgical treatment of pediatric BPHC [18]-[21]. As these facts are well discussed in the literature which indicates that an extensive pulmonary resection is not advised inpediatric BPHC, we could conclude that minimal invasive methods are preferred in this group of patients. This principle of minimal invasive approach with minimal lung parenchymal destruction could be achieved safely by our approach.

Different types of approaches are discussed in the relevant literature for treatment of pediatric BPHC including staged surgeries by two separate posterolateral thoracotomies at 3 weeks interval, single session two posterolateral thoracotomies, median sternotomy or clamshell thoracotomy [17] [18] [20] [21]. But single session two anterolateral thoracotomies to approach both lungs and pleural cavity in the same time for pediatric BPHC are not discussed in the literature as we are reporting on a relatively large number of patients.

An important point in management of pediatric BPHC by posterolateral thoracotomy is that contralateral lung should be protected and the ipsilateral lung should be collapsed to facilitate the operation but in pediatric BPHC the main problem is that no lung can be labeled as healthy. Moreover single lung ventilation of the non-operative side can lead to various complications including atelectasis, hypoxemia, rupturing of the other side cyst [2] [5] [9] [11] [19]. In adults the use of double lumen tube during pulmonary hydatid surgery has solved the problem of isolating the two lungs as it is possible to control ventilation and prevent flooding of the contralateral lung in case of rupture. In pediatric patients, single lung ventilation (SLV) may be performed by advancing the endotracheal tube (ETT) into the main stem bronchus opposite the side of surgery, after which the non-ventilated lung will deflate because of absorption atelectasis. Problems can occur, however, when this technique is used [19]-[21]. If a smaller, un-cuffed ETT is used, it may be difficult to provide an adequate seal of the intubated bronchus. To solve the problem of positioning the patient and SLV it seems that our method of two anterolateral thoracotomy is the solution for this obstacle.

Median sternotomy is not an ideal approach in pediatric BPHC because the surgery will involve mediastinum which is originally not involved by the pathology and risk of seeding is there despite all measures of isolation.

Besides, in pediatric population the future necessity for cardiac surgery should be put in consideration [16] [17].

Our results are highly favoring this approach for BPHC because it has a very low morbidity and mortality rate with a relatively acceptable cosmetic scar.

\section{Conclusions}

In conclusion, pediatric BPHC must be regarded as a different clinical entity because of their early age occur- 
rence, more serious symptoms and the need for prolonged care with higher costs.

We believe that single session bilateral anterolateral thoracotomy is a better approach than either one stage successive thoracotomies, median sternotomy or clamshell thoracotomy as it involves less postoperative pain and does not precipitate a decrease in respiratory capacity.

The surgical treatment of BPH must be individually tailored in each and every case. One-stage surgery is superior to classic two-stage operations as it decreases the morbidity, hospital stay and costs.

However, larger studies and longer follow-up are necessary for better understanding the rate of recurrence in BPHC, cosmetic acceptance and long term respiratory function abnormalities of our approach.

\section{Acknowledgements}

We would like to acknowledge all our personnel who assisted in serving our patients.

\section{Conflict of Interest}

The author declares that he has no competing interests.

\section{Author's Contribution}

Aram Baram: surgeon performed the operations, study design, follow-up, data collection, statistical analysis.

\section{References}

[1] Balci, A.E., Eren, N., Eren, S. and Ulku, R. (2002) Ruptured Hydatid Cysts of the Lung in Children: Clinical Review and Results of Surgery. The Annals of Thoracic Surgery, 74, 889-892. http://dx.doi.org/10.1016/S0003-4975(02)03785-2

[2] Kuzucu, A., Soysal, O., Zgel, M.O. and Yologlu, S. (2004) Complicated Hydatid Cysts of the Lung: Clinical and Therapeutic Issues. The Annals of Thoracic Surgery, 77, 1200-1204. http://dx.doi.org/10.1016/j.athoracsur.2003.09.046

[3] Topçu, S., Kurul, I.C., Tastepe, I., Bozkurt, D., Gülhan, E. and Çetin, G. (2000) Surgical Treatment of Pulmonary Hydatid Cysts in Children. The Journal of Thoracic and Cardiovascular Surgery, 120, 1097-1101.

[4] Mirshemirani, A.R., Razavi, S. and Sadeghian, S. (2009) Surgical Treatment of Pulmonary Hydatid Cyst in 72 Children. Tanaffos, 8, 56-61.

[5] Gajbhiye, A.S., Sonak, M.M., Meshram, M.M., Kathod, A.P. and Gajaralwar, R.S. (2013) Surgical Management of Pulmonary Hydatid Cyst. IOSR Journal of Dental and Medical Sciences (IOSR-JDMS), 6, 59-64.

[6] Tantawy, I.M. (2010) Hydatid Cysts in Children. Annals of Pediatric Surgery, 6, 98-104.

[7] Pejhan, S., Zadeh, M.R.L., Javaherzadeh, M., Behgam, M., Shadmehr, Arab, M., Kakhki, A.D., Farzanegan, R. and Dezfouli, A.A. (2007) Surgical Treatment of Complicated Pulmonary Hydatid Cyst. Tanaffos, 6, 19-22.

[8] Biswas, B., Ghosh, D., Bhattacharjee, R., Patra, A., Basuthakur, S. and Basu, R. (2004) One Stage Surgical Management of Hydatid Cyst of Lung \& Liver by Right Thoracotomy \& Phrenotomy. Indian Journal of Thoracic and Cardiovascular Surgery, 20, 88-90.

[9] Karavdic, K. and Guska, S. (2011) Surgical Treatment of Pulmonary Hydatid Disease in Children-A Retrospective Study. Medical Archives, 65.

[10] Petrov, D.B., et al. (2001) Surgical Treatment of Bilateral Hydatid Disease of the Lung. European Journal CardioThoracic Surgery, 19, 918-923. http://dx.doi.org/10.1016/S1010-7940(01)00693-5

[11] Cetin, G., Dogan, R., Yuksel, M., Alp, M., Ucanok, K., Kaya, S. and Unlu, M. (1988) Surgical Treatment of Bilateral Hydatid Disease of the Lung via Median Sternotomy: Experience in 60 Consecutive Patients. The Journal of Thoracic and Cardiovascular Surgery, 36, 114-117. http://dx.doi.org/10.1055/s-2007-1020056

[12] Jakob, H., Kohlhaüfl, M., Hürter, T., Steppling, H. and Oelert, H. (1989) Echinococcal Disease of Both Lungs and Liver: Successful Simultaneous Resection. The Journal of Thoracic and Cardiovascular Surgery, 97, 640-641.

[13] Karaoglanoglu, N., Kurkcuoglu, I.C., Gorguner, M., Eroglu, A. and Turkyilmaz, A. (2001) Giant Hydatid Lung Cysts. European Journal Cardio-Thoracic Surgery, 19, 914-917. http://dx.doi.org/10.1016/S1010-7940(01)00687-X

[14] Caushi, F., Qirjako, G., Bani, I., Hatibi, A., Skenduli, I., Shima, E. and Menzelxhiu, A. (2013) How to Manage the Complicated Hydatid Cyst of the Lung? Is Any Special Surgical Procedure More Preferred? Journal of Cardiothoracic Surgery, 8, P147. http://dx.doi.org/10.1186/1749-8090-8-S1-P147 
[15] Vlad, D.C., Neghina, A.M., Dumitrascu, V., Marincu, I., Neghina, R. and Calma, C.L. (2013) Cystic Echinococcosis in Children and Adults: A Seven-Year Comparative Study in Western Romania. Foodborne Pathogens and Disease, 10, 189-195. http://dx.doi.org/10.1089/fpd.2012.1281

[16] Aslanabadi, S., Zarrintan, S., Abdoli-Oskouei, S., Salehpour, F., Zarrintan, A., Beheshtirouy, S., Abdollahi, H. and Badebarin, D. (2013) Hydatid Cyst in Children: A 10-Year Experience from Iran. African Journal of Paediatric Surgery, 10, 140-144. http://dx.doi.org/10.4103/0189-6725.115040

[17] Khanday, Z.S., Deepak, J., Bagdi, R.K., Balagopal, Agarwal, P., Madhu and Balamourougane (2009) Video-Assisted Thoracic Surgery in Children: Our Institutional Experience. Sri Ramachandra Journal of Medicine, 1, 45-48.

[18] Talaiezadeh, A.H. and Maraghi, S. (2006) Hydatid Disease in Children: A Different Pattern Than Adults. Pakistan Journal of Medical Sciences, 22, 329-332.

[19] Singh, H., Kohli, H., Grewal, A. and Budhiraja, S. (2008) Anaesthetic Considerations in a Child with Bilateral Hydatid Cysts of Lung. Indian Journal of Anaesthesia, 52, 849-852.

[20] Khalilzadeh, S., Baghaie, N., Ghafarzadeh, B., Shadmehr, M.B. and Velayati, A.A. (2004) Hydatid Pulmonary Disease in Children: A Nine-Year Study. Tanaffos, 3, 43-48.

[21] Montazeri, V., Sokouti, M. and Rashidi, M.R. (2007) Comparison of Pulmonary Hydatid Disease between Children and Adults. Tanaffos, 6, 13-18. 